\title{
Rescaling Energy? Räumliche Neuordnungen in der deutschen Energiewende
}

\author{
Sören Becker ${ }^{1,2}$ and Matthias Naumann ${ }^{3}$ \\ ${ }^{1}$ Geographisches Institut der Universität Bonn, Bonn, Germany \\ ${ }^{2}$ Integrative Research Institute on Transformations of Human-Environment Systems (IRITHESys) an der \\ Humboldt-Universität zu Berlin, Berlin, Germany \\ ${ }^{3}$ Institut für Geographische Wissenschaften der Freien Universität Berlin, Berlin, Germany \\ Correspondence to: Matthias Naumann (matthias.naumann@fu-berlin.de)
}

Received: 15 February 2017 - Revised: 9 June 2017 - Accepted: 22 June 2017 - Published: 24 July 2017

Kurzfassung. The German energy transition is not only characterized by wide technological changes but also by spatial restructuring. The decentralization of energy supply potentially increases the importance of the regional or local scale. The Anglo-American debate on the Politics of Scale addresses the production and transformation of scale while energy issues have not yet been systematically addressed. This paper combines the theoretical paradigms of scale, rescaling and scalar strategies with empirical examples from the German energy transition. Using the cases of energy regions, remunicipalizations and social movements, the implications of the German energy transition are analyzed regarding the role of scale. The paper argues that the perspective of the Politics of Scale can contribute not only to a better understanding of the different dimensions of energy transitions but also to critical energy geographies in general.

\section{Einleitung}

Die Transformation der Energieversorgung hin zu erneuerbaren Energien ist mit weitgehenden lokalen und regionalen Veränderungen verbunden. Stichworte wie dezentrale Energiesysteme in ländlichen Räumen (Gailing und Röhring, 2015), die Entwicklung von Energieregionen (Späth und Rohracher, 2010), die Gründung von Bioenergiedörfern (von Bock und Polach et al., 2015), Rekommunalisierungen städtischer Energieversorger (Becker et al., 2016) oder BürgerInnen-Energie bzw. Community Energy (Radtke, 2016; Seyfang et al., 2013) stehen für eine Entwicklung hin zu kleinteiligeren, stärker als bisher lokal organisierten Formen der Energieversorgung. Damit verbunden sind Fragen von Standorten von Anlagen (Bosch et al., 2016) und deren Akzeptanz (Stegert und Klagge, 2015), neue Formen regionaler Governance (Bauriedl, 2016), aber auch spezifische föderale Verteilungswirkungen (Monstadt und Scheiner, 2016) oder die Integration europäischer Netze (Fromme, 2016). Häufig wird dabei auf die Rolle unterschiedlicher Akteure - wie etwa Versorgungsunternehmen, Umwelt- und Unternehmensverbände, soziale Bewegungen und Initiativen für BürgerInnenEnergie, Instanzen der Raumplanung, Kommunen und weitere staatliche Institutionen - verwiesen, die auf verschiedenen Ebenen als Treiber oder Opponenten der Energiewende auftreten können (Bauriedl, 2016; Klagge, 2013).

Darüber hinaus versuchen Arbeiten aus der angloamerikanischen Humangeographie, aktuelle Veränderungen in der Energieversorgung mit grundlegenden theoretischen Paradigmen der Disziplin zu verknüpfen (Bridge et al., 2013; Calvert, 2016; Huber, 2015). Sowohl Calvert (2016) als auch Huber (2015) weisen dabei auf das Potential hin, gegenwärtige Veränderungen im Energiesektor als Raumproduktionen zu diskutieren. Die Frage räumlicher Maßstabsebenen (Scales), deren Entstehung und Veränderung, spielt dabei eine zentrale Rolle. Während Bridge et al. (2013:337) Scale als die Größe oder die räumliche Ausdehnung von energiebezogenen Phänomenen verstehen, greifen wir den Hinweis von Huber und Calvert auf und bringen die Veränderungen der deutschen Energiewende in Zusammenhang mit der Diskussion um die Politics of Scale und Rescaling. 
Damit möchten wir zwei Debatten miteinander verknüpfen: erstens die Debatte um Scale in der angloamerikanischen kritischen Humangeographie, die mit dem Begriff die Produktion und Dynamik räumlicher Maßstabsebenen sozialen Handelns beschreibt und skalare Strategien von Akteuren in den Blick nimmt. Zweitens beziehen wir uns auf die stetig wachsende Literatur zu den räumlichen Implikationen der deutschen Energiewende, die ein aktuelles Beispiel für soziotechnische Transformationen, deren Möglichkeiten und Widersprüche darstellt. Wir fragen nach den Potentialen der Scale-Debatte für umweltpolitische Themen insgesamt und energiegeographische Fragestellungen im Besonderen und erörtern, welche skalare Dimensionen die bundesdeutsche Energiewende aufweist. Dabei stützen wir uns auf eigene Forschungsprojekte zur deutschen Energiewende, die wir als empirische Beispiele zur Illustration heranziehen. Der Beitrag gliedert sich wie folgt: zunächst geben wir einen Überblick über die humangeographische ScaleDebatte, deren Hintergründe, zentrale Begriffe und aktuelle Entwicklungen. Anschließend diskutieren wir ausgewählte Aspekte der deutschen Energiewende hinsichtlich ihrer räumlichen Maßstäblichkeit und der skalaren Strategien verschiedener Akteure, wie etwa Versorgungsunternehmen, soziale Bewegungen, Kommunen und weitere staatliche Institutionen. Im Fazit geben wir einen Ausblick auf mögliche Anschlüsse für eine geographische Energieforschung aus einer Scale-Perspektive und formulieren weiteren Forschungsbedarf.

\section{Scale, Rescaling und die Politics of Scale - Theoretische Hintergründe und Entwicklungslinien einer Debatte}

Seit knapp 30 Jahren spielt der Begriff Scale eine zentrale Rolle in der angloamerikanischen kritischen Humangeographie (Brenner, 2004; Jonas, 2006; MacKinnon, 2011). Ausgangspunkt der Scale-Debatte ist, dass die Produktion von Raum und räumlichen Maßstabsebenen untrennbar mit gesellschaftlichen Verhältnissen verbunden ist. Die ScaleDebatte steht damit in der Tradition der Raumtheorien von Henri Lefebvre, David Harvey und Edward Soja. Während Multi-Level-Governance in der Politikwissenschaft (Benz, 2007) auf die Interaktion und die komplexe Steuerung zwischen verschiedenen räumlichen Ebenen fokussiert, nimmt die Scale-Debatte in der Humangeographie in den Blick, wie räumliche Maßstabsebenen entstehen und mit gesellschaftlichen Machtverhältnissen und Prozessen ungleicher Entwicklung verbunden sind. Insbesondere neuere Forschungsarbeiten zur Governance von Umwelt und natürlichen Ressourcen, die sozialräumliche und politische Maßstabsebenen in Verbindung zur Materialität von Natur und natürlichen Ressourcen setzen, bieten Anschlüsse für die geographische Energieforschung. In diesem Kapitel führen wir kurz in Grundkonzepte der Scale-Debatte und deren Anwendung auf Umweltfragen ein. Wir betrachten, wie Scales als produzierte räumliche Strukturen zu verstehen sind, das State Rescaling als Veränderung staatlicher Organisation und die skalaren Strategien unterschiedlicher Akteure.

Die Debatte um Scale hat ihren Ursprung in der räumlichen Weiterentwicklung politökonomischer Theorien Anfang der 1980er Jahre. AutorInnen, wie etwa Immanuel Wallerstein, interpretieren räumliche Strukturen als Ergebnis gesellschaftlicher Kräfteverhältnisse, wie zum Beispiel internationaler Abhängigkeiten von Entwicklungs- und Schwellenländern (Wallerstein, 2004). Das Entstehen dieser Strukturen wird auf einen zunehmend globalisierten Kapitalismus zurückgeführt, der fortwährend neue Ungleichheiten produziert. In seinem Buch „Uneven Development“ entwickelte Neil Smith (2008[1984]) eine Theorie über das Verhältnis zwischen Prozessen der Kapitalakkumulation und der Produktion von Räumen im Allgemeinen und von verschiedenen räumlichen Ebenen im Besonderen. Letztere entstehen vor allem aus der Notwendigkeit, dass Kapital gleichzeitig lokal verankert und mobil zwischen den verschiedenen Orten bleiben muss. Die entstehenden Strukturen - Unternehmensverflechtungen, Märkte, verschiedene Ebenen von staatlichen Organisationen und Regulation - werden als ,scalar fix“ bezeichnet. Dieser leitet sich in der klassischen Interpretation aus den Kapitalinteressen ab, wird jedoch durch gesellschaftliche Kämpfe kontinuierlich verschoben und beeinflusst (Antentas, 2015).

Während diese klassische Interpretation von Scale sehr stark in einer marxistischen Tradition verwurzelt war, wurde sie im Laufe der Debatte zum Gegenstand poststrukturalistischer Kritik (Marston et al., 2005). Diese Kritik unterstellte der bisherigen Scale-Theorie ein essentialistisches Verständnis, das die Wirkung von Scale als existierende Struktur nicht hinterfragt. Aufbauend auf dieser Kritik hat sich auch in der marxistisch geprägten Konzeption von Scale ein Verständnis durchgesetzt, dass Scales nicht unabhängig von gesellschaftlichen Prozessen erfasst werden können, in die sie eingebunden sind. Scale sollte damit weniger als bestehende Struktur analysiert werden, sondern als Produkt gesellschaftlicher Auseinandersetzungen und skalarer Praxen (MacKinnon, 2011).

Ein Bereich, in dem die Veränderung von Scales umfassend untersucht wurde, ist die Verschiebung staatlicher Befugnisse im Zuge neoliberaler Reformen der 1980er und 1990er Jahre. Das dort beobachtete Zusammenspiel von Prozessen der Internationalisierung, der Entmachtung bestehender politischer Maßstabsebenen und die Emergenz neuer, häufig wettbewerblicher Governance-Formen auf subnationaler Ebene wird als State Rescaling bezeichnet (Brenner, 2004). Die Restrukturierung des Staates ist ein ,zutiefst herrschaftsförmiger Prozess" (Wissen, 2008:9), der wiederum die Bedingungen künftigen Handelns verschiebt. Ein Beispiel hierfür ist die Abschaffung der von der Labour-Partei dominierten Regionen in England durch Margaret Thatcher (Wissen, 2008). Es werden aber auch Strategien wie die ge- 
zielte Förderung von Metropolregionen (Pütz, 2005) oder die Ausweitung der Kompetenzen der Europäischen Union in diesem Zusammenhang untersucht (Jessop, 2009). Durch State Rescaling verändern sich damit sowohl die Kompetenzen verschiedener staatlicher Organisationsebenen für einzelne Politikfelder als auch der Zugang zu diesen Institutionen (MacKinnon, 2011). Diese Veränderungen sind kein Selbstzweck, sondern dienen der Durchsetzung politischer Interessen.

Scales und scalar fixes sind das Produkt gesellschaftlicher Auseinandersetzungen zwischen unterschiedlich mächtigen Akteuren und deren politischen Praxen. Beim Blick auf diese Auseinandersetzungen werden verschiedene skalare Strategien in den Blick genommen: der Versuch, die Konstruktion und Bedeutung von Scales bewusst zu beeinflussen, aber ebenso der Versuch, gewisse Maßstabsebenen zu ,überspringen“, um die eigenen Interessen auf einer anderen Ebene besser durchsetzen zu können. Dieses Konzept des Scale Jumping geht auf Neil Smith (1993) zurück, der in seiner Untersuchung zum „homeless vehicle“ zeigt, wie ein von KünstlerInnen entwickeltes unmotorisiertes Fahrzeug New Yorker Obdachlosen hilft, den räumlichen Radius bzw. den Scale ihrer Aktivitäten von einem Stadtviertel auf fast die gesamte Stadt zu erweitern. Scale Jumping ist damit eine Strategie, den räumlichen Bezugsrahmen des eigenen Handelns zu erweitern. Dies kann die bestehenden Macht- und Herrschaftsverhältnisse verändern (Smith, 2000:726) und auch bislang ausgeschlossenen Akteuren einen neuen Handlungsspielraum aufschließen. Skalare Strategien werden sowohl von Unternehmen angewendet (Chapura, 2009) als auch von Gewerkschaften (Heeg, 2014) und sozialen Bewegungen (Antentas, 2015) eingesetzt. Die Fähigkeit, die Reichweite des eigenen politischen Handelns zu verändern, wird damit selbst zu einer Machtressource, die aufgrund von Mobilität, institutioneller Rahmenbedingungen und Zugang zu Netzwerken oder Wissen ungleich verteilt ist (Adger et al., 2005).

Neben politökonomischen Anwendungsfeldern der ScaleTheorie wurde das Konzept in den letzten Jahren auf das Feld der Governance von Umwelt-und natürlichen Ressourcen übertragen (Bulkeley, 2005; Cohen und Bakker, 2014; Reed und Bruynell, 2010). Ausgangspunkt dieser Arbeiten ist häufig die fehlende Übereinstimmung zwischen politischadministrativen und naturräumlichen Maßstabsebenen, beispielsweise bei Wasserscheiden oder Nationalparks (Cohen und McCarthy, 2015). Goodman et al. (2008) betonen zudem, dass Politics of Scale in Umweltkonflikten eine große Rolle spielen, Norman et al. (2012) bringen das gleiche Argument für die Regulation von Wasserressourcen. Wie Swyngedouw (2007) am Beispiel der Einführung von Flusseinzugsgebieten in der spanischen Wasserregulation zeigt, sind die Zuschnitte scheinbar ,natürlicher“ Räume ein Resultat politischer Interessen. In die Wahrnehmung und Nutzung von Natur sind gesellschaftliche Machtverhältnisse eingeschrieben, zum Beispiel in Form des ungleichen Zugangs zu Ressourcen (Harvey, 1996). Cohen und Bakker führen hier den Begriff des ,eco-scalar fix“" ein, der die skalare Ordnung natürlicher Ressourcen als Ausdruck gesellschaftlicher Kräfteverhältnisse versteht (2014:132).

Das Bestreben, natürliche Ressourcen zu kontrollieren oder in Wert zu setzen, führt zu Auseinandersetzungen um skalare Ordnungen (Swyngedouw und Heynen, 2003:913). Das wird zum Beispiel bei der Sicherstellung von Anbauflächen von Agrarkraftstoffen in Südamerika (Dietz et al., 2015) oder von Palmöl in Indonesien (Brad, 2016) sichtbar, wo jeweils verschiedene Akteure (KleinbäuerInnen, internationale Unternehmen, NGOs, staatliche Stellen) versuchen, ihre Interessen auf verschiedenen Ebenen durchzusetzen und die strukturelle Bedeutung der verschiedenen politischen Ebenen zu verändern. In Diskussionen um Rescaling der Governance von Umwelt und natürlichen Ressourcen wird entsprechend auf neue räumliche Zuschnitte und Maßstabsebenen sowie auf die grundlegende Veränderung der Rolle des Staates verwiesen. So stellen Reed und Bruynell (2010) Prozesse des „Scaling Up“ und des „Scaling Down“ fest, in denen die Verantwortung für die Regulation von natürlichen Ressourcen auf höhere bzw. niedrigere Scales übertragen wird. Für Cohen und McCarthy (2015) sind diese Prozesse des Rescalings Ausdruck einer neoliberalen Umstrukturierung. Oft werden Entscheidungen auf nicht demokratisch legitimierte Maßstabsebenen verlagert (ebd.:19). Zudem besteht die Gefahr, durch eine Lokalisierung der Zuständigkeiten und Ressourcen strukturelle Ungleichheiten zwischen Orten und Regionen zu reproduzieren (ebd.:6). Somit bringt die Reskalierung der Governance natürlicher Ressourcen GewinnerInnen und VerliererInnen hervor.

Ein weiterer Aspekt des Rescalings sind skalare Strategien, die von unterschiedlichen Akteuren für die Durchsetzung ihrer Interessen in der Environmental Governance eingesetzt werden (Köhler, 2008:212). Beispiele hierfür sind die auf lokaler wie auch internationaler Ebene agierenden städtischen Verwaltungen in Städtenetzwerken (Bulkeley, 2005), aber auch Bewegungen für Klimagerechtigkeit, die sowohl auf internationalen Gipfeln als auch mit lokalen dezentralen Aktionen vertreten sind (Chatterton et al., 2013). Hoogesteger und Verzijl (2015) zeigen anhand der Kämpfe ekuadorianischer und peruanischer KleinbäuerInnen um den $\mathrm{Zu}$ gang zu Wasserressourcen, wie indigene Bevölkerungsgruppen skalare Strategien einsetzen. Der Begriff der „grassroots scalar strategies" steht dafür, dass die lokalen Bewegungen auf verschiedenen Scales Allianzen eingehen, um ihrer Position Gehör zu verschaffen (ebd.:15).

Insgesamt haben sowohl die Scale-Debatte als auch Arbeiten zur Governance von Umwelt und natürlichen Ressourcen in konzeptioneller und empirischer Hinsicht von einer skalaren Perspektive auf Umweltpolitik profitiert. Dabei lassen sich drei Dimensionen der Skalierung von Mensch-UmweltVerhältnissen identifizieren: Erstens die materielle Dimension, etwa bei Anlagen und Netzen der Wasserversorgung, zweitens die institutionelle Dimension, zum Beispiel bei der 
Frage nach dem Zugang zu natürlichen Ressourcen und drittens die diskursive Dimension, beispielsweise wie lokal oder global eine Ressource von unterschiedlichen Interessengruppen angesehen wird. Im Gegensatz zur mittlerweile recht umfangreichen Literatur zur skalaren Dimension von natürlichen Ressourcen und deren Regulation gibt es bislang aber erst wenige Ansätze, die Scale-Debatte auf Energie zu beziehen. Im folgenden Kapitel werden daher die Möglichkeiten untersucht, energiegeographische Fragen sowie Aspekte der bundesdeutschen Energiewende aus einer Scale-Perspektive zu betrachten.

\section{Scale und Energiewende - Mögliche Anschlüsse und empirische Beobachtungen}

Energieinfrastrukturen sind soziotechnische Systeme, die verschiedene räumliche Maßstabsebenen miteinander verbinden. Während Hoch- und Höchstspannungsnetze den Austausch von Elektrizität über weite Strecken ermöglichen, bilden regionale und lokale Verteilnetze auf Mittelspannungsebene den Übergang zu den verbrauchernahen Niederspannungsnetzen. Netze und Kraftwerke werden von Unternehmen, die auf unterschiedlichen räumlichen Ebenen aktiv sind - vom klassischen Stadtwerk bis zum multinationalen Konzern - betrieben. Neben diesen technischen und ökonomischen Skalierungen bestehen in Deutschland verschiedene Ebenen der Regulation, von Bundesgesetzen, der Bundesnetzagentur, bis hin zu regionalen und lokalen Energiewende- und Klimaschutzkonzepten. Unser Ziel ist es, im Folgenden bestehende räumliche Ebenen zu hinterfragen und Ansatzpunkte aufzuzeigen, wie die deutsche Energiewende im Strombereich selbst zu einer Veränderung dieser skalaren Strukturen beigetragen hat. Vor diesem Hintergrund diskutieren wir erstens vorliegende Arbeiten zur skalaren Dimension des Energiesektors und dessen Veränderungen, zweitens illustrieren wir mit einigen empirischen Beispielen die engen Verknüpfungen der Energiewende mit dem Wandel räumlicher Maßstabsebenen und drittens diskutieren wir die Ambivalenzen dieser Reskalierungen hinsichtlich der Frage, inwieweit die räumlichen Neuordnungen im Energiesektor einen emanzipatorischen Charakter haben bzw. im Zusammenhang mit neoliberalen Projekten stehen.

\subsection{Energie als blinder Fleck der Scale-Debatte, Scale als Thema der Energiegeographie}

Während das Scale-Konzept in Forschungen zur Governance von Umwelt und natürlichen Ressourcen mittlerweile eine rege Debatte angestoßen hat, ist die Diskussion zum Energiebereich weniger weit fortgeschritten. In den vorliegenden Arbeiten zum Rescaling des Energiesektors lassen sich drei Zugänge unterscheiden. Erstens die Diskussion um die Maßstäblichkeit von Anlagen zur Stromerzeugung und deren Dezentralisierung; zweitens die Rolle von räumlichen Maßstäben bei Transformationen zur Nachhaltigkeit und drit- tens erste Arbeiten zur Verschiebung von räumlichen Ebenen in der Energie- und Klimapolitik. Bislang bezieht sich nur der dritte Zugang explizit auf die oben dargestellte kritische Scale-Forschung.

„Dezentralisierung“ hat sich zu einem Kernbegriff in Diskussionen um die Energiewende entwickelt (Eiselt, 2012). Darunter werden zwei Phänomene verstanden: zum einen die Verkleinerung von Anlagen der Strom- und Wärmeerzeugung, zum anderen eine räumliche Verlagerung der Standorte, auch dadurch bedingt, dass es mehr Anlagenstandorte gibt. Diese Verschiebung von ,energy for space“ zu „energy from space“ (Brücher, 2009) ist jedoch analytisch schwierig zu erfassen: Großprojekte wie Windparks sowohl an Land als auch offshore, flächenintensive Solarparks etc. können den grundsätzlich dezentralen Charakter der Nutzung erneuerbarer Energieträger in Frage stellen. Insofern sprechen Gailing und Röhring (2015) von einem Spannungsfeld zwischen zentralen und dezentralen Strukturen, während Bauriedl (2016) untersucht, wie neue lokale Kooperationen in bestehende hierarchische Steuerungsformen auf verschiedenen Maßstabsebenen eingebettet werden. Klagge (2013) spricht von einer „Dualität“" von auf der Bundesebene angesiedelten ökonomischen Anreizen einerseits und auf der lokalen bzw. regionalen Ebene angesiedelten raumplanerischen Einflussmöglichkeiten andererseits. Bosch et al. (2016) diskutieren die Raumverträglichkeit von Anlagen erneuerbarer Energien als eine wichtige Herausforderung bei der Umsetzung der Energiewende. Die räumlichen Verschiebungen und veränderten Maßstäblichkeiten innerhalb der Energiewende sind damit bereits ein etabliertes Thema der geographischen Energieforschung, jedoch fehlt bisher eine Verbindung mit den dahinterliegenden Politics of Scale.

Dieses Defizit betrifft auch die Debatte zur Geographie von Transitionen zur Nachhaltigkeit (Sustainability Transitions) (Coenen et al., 2012). Diese entwickelte sich aus einer evolutionären Perspektive auf Innovationen und den Wandel soziotechnischer Systeme (Geels, 2002). In ihrem Zentrum steht die „Multi-Level-Perspektive“, die davon ausgeht, dass Innovationen zuerst in kleinräumigen Nischen entstehen und dort erprobt werden, bevor sie bestehende Strukturen eines soziotechnischen Regimes beeinflussen oder ablösen können. Um das Entstehen von Nischen und das $\mathrm{Zu}$ sammenwirken von Nischen und Regimes hat sich eine sehr breite Literatur entwickelt (z.B. Geels und Schot, 2007), die jedoch wegen ihrer fehlenden räumlichen Präzisierung kritisiert wurde (Lawhon und Murphy, 2012). Beispielsweise wurde häufig angenommen, dass eine Nische als lokal gilt, das Regime jedoch auf der nationalen Ebene angesiedelt wird. Infolgedessen hat sich eine Diskussion um eine geographische Vertiefung dieser Ansätze entwickelt, die auch räumliche Maßstabsebenen thematisiert (Raven et al., 2012). Coenen et al. (2012) sehen im Begriff von Scale einen naheliegenden Zugang für dieses Anliegen: „In short that the multilevel perspective could also become a multi-scalar perspective“ (ebd.:972). Inspiriert von der relationalen Wirtschafts- 
geographie, wird hier Scale vor allem über Nähe interpretiert. Soziale Beziehungen zwischen Akteuren, Organisationen, Wissensbeständen etc. bilden räumliche Netzwerke aus, die verschiedene Territorien durchschneiden können und deren Ausmaß als Begrenzung der jeweiligen Scales verstanden wird (Raven et al., 2012). Dadurch sollen Nischen und Regimes räumlich begrenzt darstellbar werden. Diese Perspektive trägt zwar der sozialen Konstruktion verschiedener räumlicher Maßstäbe Rechnung, nimmt jedoch die politischen Prozesse der Entstehung dieser Scales nicht in den Blick.

Im Gegensatz dazu stehen Arbeiten, die sich explizit auf die gesellschaftliche Konstruktion und Aushandlung von räumlichen Maßstabsebenen beziehen, erst am Anfang. Osofsky (2011) untersucht das Entstehen und $\mathrm{Zu}-$ sammenwirken verschiedener politischer Ebenen in der USamerikanischen Klimapolitik. Cowell et al. (2015) beziehen sich auf die Übertragung von Verantwortung auf die Teilstaaten des Vereinigten Königreichs, was zu neuen regionalen Strategien und Handlungsräumen geführt hat. Während der regionale Ausbau von erneuerbaren Energien stark von gesamtstaatlichen Förderstrategien abhängt, werden Fragen der Akzeptanz von Anlagen weiterhin auf kleinräumiger Ebene entschieden. Dahinter steht die Beobachtung einer zunehmenden Komplexität von Energiepolitik, die mit neuen Governance-Modi auf unterschiedlichen Ebenen einhergehen kann. Ähnlich verfolgen Bouzarovski et al. (2015) die Frage, wie durch Umstrukturierungen auf dem europäischen Gasmarkt neue technische und institutionelle Räume entstehen. Unser Ziel im nächsten Abschnitt ist es daher, die skalare Komplexität der Energiewende in Deutschland darzustellen.

\subsection{Politics of Scale der deutschen Energiewende}

Insgesamt ist die Energiewende von einer multiskalaren Dynamik geprägt. Die erfolgreiche Realisierung der bundespolitischen Beschlüsse zur Energiewende hängt einerseits ganz maßgeblich von lokalen und regionalen Projekten ab, über deren politische und rechtliche Rahmenbedingungen jedoch andererseits auf Bundesebene entschieden wird. Gleichzeitig müssen die Rahmenbedingungen der Energiewende in Deutschland mit den Bestrebungen kompatibel sein, einen europäischen Energiemarkt zu schaffen (Haas, 2016). Die veränderten Politics of Scale der deutschen Energiewende stellen wir anhand von drei Beispielen dar: das Entstehen neuer Handlungsräume, Rekommunalisierungen in der Energieversorgung und skalare Strategien energiepolitischer Akteure. Hierbei geht es weniger um eine systematische Darstellung von empirischen Untersuchungen, als vielmehr um Anregungen für die weitergehende Untersuchung der skalaren Dimension der Energiewende.

Die technologische Verschiebung von einer zentralistischen und auf fossilen Energieträgern beruhenden hin zu einer stärker dezentral und erneuerbar geprägten Energieversorgung wird durch das Entstehen neuer Handlungsräume und Maßstabsebenen begleitet (Gailing und Röhring, 2015). Ein Beispiel hierfür sind Energieregionen, die durch regionale Kooperationen und Diskurse konstruiert werden (Späth und Rohracher, 2010). Der räumliche Zuschnitt dieser Regionen variiert, sie können einen oder mehrere Landkreise umfassen, aber auch deutlich kleiner dimensioniert sein. Die verschiedenen Projekte haben gemeinsam, dass die Region als zentrale Maßstabsebene für eine nachhaltige Transformation der Energieversorgung identifiziert wird. Mit dem Ziel, das Energiesystem durch neue Erzeugungsanlagen und auch veränderte Wertschöpfungsketten regional zu gestalten, werden neue Organisationen für die Kooperation zwischen verschiedenen Akteuren gegründet (Späth und Rohracher, 2014). Die Region wird dadurch als neue Handlungsebene für die Energieversorgung konstruiert, was sich auch im Selbstverständnis dieser Regionen und in einer neuen regionalen Identität widerspiegeln kann. Gleichzeitig können dadurch andere Identitäten, zum Beispiel als Tourismusregion, abgewertet werden.

Mit dem Ausbau erneuerbarer Energieträger sind aber auch weitere neue Maßstabsebenen verbunden. So stellen die von der Regionalplanung ausgewiesenen „Windeignungsgebiete" eine neue Ebene dar, für die die künftige Gestaltung der Energieversorgung verhandelt wird (Klagge, 2013). Darüber hinaus definieren sich Kommunen oder Ortsteile als Bioenergiedörfer (von Bock und Polach et al., 2015) bzw. als energieautarke Gemeinden. Das beinhaltet materielle Veränderungen, indem z.B. versucht wird, im Gemeindegebiet durch neu gebaute Anlagen so viel Strom oder Wärme zu produzieren wie dort verbraucht wird oder gar in sich geschlossene „Inselnetze“ aufzubauen. Im Sinne der angestrebten Steigerung der Energieeffizienz wird aber auch die Ebene städtischer Quartiere in der Energieversorgung wichtiger. Für Stadtteile und -quartiere werden Maßnahmen der energetischen Sanierung und teilweise auch eigene Versorgungskonzepte geplant (Freytag et al., 2014). Private Haushalte, die als „ProsumentInnen“ von Strom- und WärmeverbraucherInnen zu ErzeugerInnen werden können, stellen ebenfalls eine neue räumliche Ebene von Versorgungsstrukturen dar.

Eine Verschiebung der institutionellen und räumlichen Ordnung der deutschen Energieversorgung stellen die Gründung bzw. der Rückkauf von vormals privatisierten Stadtund Regionalwerken durch Kommunen dar. Hier verändern sich sowohl die Eigentumsstrukturen als auch die Verteilung von staatlichen Einflussmöglichkeiten auf die Energieversorgung. Rekommunalisierungen gab es in Großstädten wie Hamburg oder Stuttgart, in kleineren Städten vor allem im Südwesten des Landes, in Nordrhein-Westfalen und in der Region Hamburg, bis hin zu peripheren Regionen wie dem Norden Hessens (Lormes, 2016). Es gibt verschiedene Formen von Rekommunalisierungen und Wege, wie diese realisiert werden können (Bauer, 2012). Unter Rekommunalisierungen kann ein Wandel hin zu kommunalen Eigentumsverhältnissen im Energiesektor, darunter die Integration eines neuen Teilnetzes in kommunale Netzstrukturen, die Inte- 
gration eines neuen oder bestehenden Energieversorgungsunternehmens in bereits bestehende Stadtwerke, die Neugründung eines kommunalen Energieversorgungsunternehmens oder kommunalen Netzbetreibers oder die Übernahme von Netzkonzessionen und die Erhöhung des kommunalen Gesellschaftsanteils an gemischtwirtschaftlichen Unternehmen verstanden werden (Becker et al., 2012:26f.). Wichtig ist, dass neben den Unternehmen als Organisation auch deren materielle Werte wie Netze und Kraftwerke in den Besitz der Kommune übergehen (Bauer, 2012).

Rekommunalisierungen nehmen die Prozesse der Marktkonzentration und der Privatisierung der Energieversorgung zurück, die im Zuge der Liberalisierung in den 1990er und den frühen 2000er Jahren entstanden (Bontrup und Marquardt, 2010). Häufig artikulierten städtische soziale Bewegungen die Unzufriedenheit mit den privaten Unternehmen im Energiesektor (Becker et al., 2016). Kampagnen wie „Unser Hamburg - Unser Netz“ eröffnen einen Diskurs, der sich neben dem Ziel der öffentlichen Aneignung von Energieinfrastrukturen auf eine Lokalisierung der Energieversorgung bezieht. Befindet sich ein Energieversorgungsunternehmen wieder in der Hand einer oder mehrerer Kommunen, kommen diesen auch die Gewinne aus dem Energieversorgungsunternehmen wieder zugute, mit denen andere städtische Infrastrukturdienstleistungen finanziert werden können. Hinzu kommt das erhöhte Steueraufkommen durch den lokalen Firmensitz (Reck, 2012). Ebenso können die Kommunen als neue Eigentümer die Unternehmensstrategien bestimmen und so die Stadtwerke als ein Werkzeug für den Ausbau erneuerbarer Energien nutzen (Klagge und Brocke, 2012). Rekommunalisierungen verändern EnergieGovernance: weg von einer Prägung durch private, häufig überregionale und internationale Akteure hin zu einer größeren Bedeutung des lokalen Staats. Die „Wiederentdeckung“ kommunaler Versorgungsunternehmen steht damit für einen Bedeutungsgewinn der städtischen Ebene in der Energieversorgung.

Drittens setzen verschiedene Interessengruppen bei der Aushandlung der bundesdeutschen Energiewende gezielt skalare Strategien ein, indem sie auf unterschiedlichen Maßstabsebenen aktiv sind. Dazu zählen z.B. Stadtverwaltungen, die sich in internationale Verbünde oder Organisationen zu Themen der Energieversorgung und des Klimaschutzes einbringen, und Bundesländer, die ihre Interessen im Bundesrat vertreten (Monstadt und Scheiner, 2016). Andere Beispiele sind Energiegenossenschaften, die bundesweit um Einlagen werben, um lokale Netze, wie etwa in Berlin, zu übernehmen oder eigene Erzeugungskapazitäten aufzubauen. Bei den Auseinandersetzungen um die Rahmenbedingungen für die Förderung von erneuerbaren Energien, allen voran die Einführung und Novellen des ErneuerbareEnergien-Gesetzes, bringen sich sowohl Umwelt- als auch Unternehmensverbände ein (Klagge, 2013). Gleichzeitig versuchen aber auch nationale oder internationale Energiekonzerne, ihre Anteile an kommunalen lokalen oder regionalen
Unternehmen zu behalten bzw. auszubauen. Die bisherigen „großen Vier" der deutschen Energiewirtschaft versuchen, sich durch haushaltsbezogene Dienstleistungen, wie z.B. die Installation von Solaranlagen, neue Geschäftsfelder zu erschließen. Darüber hinaus verfolgen sie ihre Interessen durch Einflussnahme auf europäischer Ebene oder durch die Anrufung internationaler Schiedsgerichte (Haas, 2016).

Aber auch zivilgesellschaftliche Initiativen wie die Protestbewegung gegen Windräder oder die Klimabewegung, die Aktionen gegen Braunkohletagebaue organisiert, wenden multi-skalare Strategien an. So sind die zahlreichen Proteste gegen die Planung, Errichtung und Erweiterung von Windkraftanlagen einerseits ein lokales Phänomen, bei dem über einzelne Standorte gestritten wird. Andererseits sind die lokalen Initiativen in der Lage, ihren Protest durch regionale, bundesweite (,Initiative Vernunftkraft“) oder internationale Zusammenschlüsse (,European Platform Against Windfarms") auf anderen Maßstabsebenen zu thematisieren. Anti-Windkraft-Initiativen versuchen, die Regionalplanung und die politischen Strategien der Bundesländer zu beeinflussen. Damit wurden aus lokalen BürgerInneninitiativen regionale: in Brandenburg initiierte der Zusammenschluss „Volksinitiative Rettet Brandenburg“ im Jahr 2016 ein landesweites Volksbegehren, in Mecklenburg-Vorpommern beteiligte sich die Partei „Freier Horizont“, hervorgegangen aus lokalen BürgerInneninitiativen gegen Windkraftanlagen, an der Landtagswahl im September 2016. Die Klimabewegung hingegen mobilisiert im Rahmen von internationalen Camps wie den Klimacamps in der Lausitz und im Rheinland zu Aktionen zivilen Ungehorsams gegen einzelne Anlagen wie Braunkohletagebaue, um international aufgestellte fossile Energieunternehmen unter Druck zu setzen. Ihr Ziel ist dabei, durch lokale Aktionen in nationale und globale energie- und klimapolitische Auseinandersetzungen zu intervenieren (Sander, 2016). Neben der Herausbildung neuer und dem Wandel bestehender Scales besteht ein dritter Aspekt des Rescalings darin, dass Akteure unterschiedliche räumliche Maßstabsebenen strategisch für die Durchsetzung ihrer Interessen nutzen.

Energieregionen, Rekommunalisierungen sowie die Strategien unterschiedlicher sozialer Bewegungen zeigen beispielhaft die Bedeutung von Scale im Kontext der bundesdeutschen Energiewende. Wie im Ausblick von Kapitel 2 angeführt, sind Fragen von Scale eng mit materiellen, institutionellen und diskursiven Veränderungen verknüpft. Für die Anlagen und Netze sowie für die politische Steuerung des Energiesystems und den damit verknüpften Diskursen spielen die jeweiligen räumlichen Maßstabsebenen eine zentrale Rolle. Tabelle 1 fasst diese verschiedenen Dimensionen eines Rescalings im deutschen Energiesektor zusammen. Die Tabelle zeigt aber auch, dass die Energiewende und die mit ihr verbundenen skalaren Verschiebungen ein noch laufender Prozess mit offenem Ausgang sind. Rescaling in der deutschen Energiewende kann also einerseits emanzipatorischen Charakter haben, indem es bislang benachteiligte Positionen 
Tabelle 1. Dimensionen der Rescaling-Prozesse in der deutschen Energiewende. (Quelle: Eigene Darstellung)

\begin{tabular}{l|l|l|l}
\hline & Beispiel Energieregionen & Beispiel Rekommunalisierungen & $\begin{array}{l}\text { Beispiel Proteste gegen Wind- } \\
\text { kraft oder Kohle }\end{array}$ \\
\hline Materielle Dimension & $\begin{array}{l}\text { Regionalisierung von Versor- } \\
\text { gungsstruktur und Wertschöp- } \\
\text { fungsketten }\end{array}$ & $\begin{array}{l}\text { Übertragung von Eigentum an } \\
\text { Netzen und Anlagen an öffentli- } \\
\text { che, eher lokale Akteure }\end{array}$ & $\begin{array}{l}\text { Konflikte um lokale Standorte } \\
\text { von Anlagen und Netzen vs. } \\
\text { Konflikte um fossile Energie- } \\
\text { wirtschaft }\end{array}$ \\
\hline Institutionelle Dimension & $\begin{array}{l}\text { Stärkung der Region als Hand- } \\
\text { lungsraum für Koordination und } \\
\text { Kooperation im Energiesektor }\end{array}$ & $\begin{array}{l}\text { Stärkung der lokalen Ebene in } \\
\text { der Energie-Governance }\end{array}$ & $\begin{array}{l}\text { Regionalisierung lokaler Pro- } \\
\text { teste vs. lokale Proteste als Re- } \\
\text { aktion auf globalen Klimawan- } \\
\text { del }\end{array}$ \\
\hline Diskursive Dimension & $\begin{array}{l}\text { Schaffung bzw. Veränderung re- } \\
\text { gionaler Identitäten }\end{array}$ & $\begin{array}{l}\text { Bestimmung gesellschaftlicher } \\
\text { Aneignung und lokaler Kontrolle }\end{array}$ & $\begin{array}{l}\text { Aushandlung von verschiede- } \\
\text { nen Vorstellungen von Land- } \\
\text { schaft und Ländlichkeit vs. Zu- } \\
\text { kunft des globalen Energiesys- } \\
\text { tems }\end{array}$ \\
\hline
\end{tabular}

stärkt. Andererseits sind diese Verschiebungen in Paradigmen neoliberaler Politik und Dynamiken ungleicher räumlicher Entwicklung eingebunden, wie wir im folgenden Abschnitt diskutieren werden.

\subsection{Rescaling in der Energiewende und ungleiche Entwicklung}

Die raumwissenschaftliche Literatur zur Energiewende stellt diese in engen Zusammenhang mit einer Dezentralisierung der Energieversorgung (Gailing und Röhring, 2015). Die politischen Implikationen der Regionalisierung oder Lokalisierung im Energiesektor sind jedoch noch genauer zu bestimmen. Diese Frage spiegelt die humangeographische Diskussion über lokale Alternativen zum Neoliberalismus wider. Während einige AutorInnen vor einer „local trap“ warnen, die die kleinste räumliche Einheit zwangsläufig als die beste ansieht (Brown und Purcell, 2005), plädieren Featherstone et al. (2012) für einen ,progressive localism“ als eine politische Alternative zu neoliberalen Paradigmen. So stellt sich die Frage, wie die Dezentralisierung der Energieversorgung als skalare Veränderung zu einer Reproduktion bzw. Verstärkung räumlicher Ungleichheiten beiträgt.

Erstens wohnt Vorstellungen eines Lokalismus der Energieversorgung die Gefahr inne, Dynamiken bestehender Machtverhältnisse und ungleicher räumlicher Entwicklung zu reproduzieren. So führte in der britischen Energiepolitik die Übertragung der energiepolitischen Verantwortung auf die Teilstaaten des Vereinigten Königreiches zu unterschiedlichen Ausbauständen und technologischen Pfaden der Energiewende (Cowell et al., 2015). Catney et al. (2014) stellen die energiepolitische Agenda in England, die lokalen Community-Projekten eine hohe Bedeutung beimisst, in den Kontext der neoliberalen Big-Society-Ideologie, die bestehende Ungleichheiten zwischen einzelnen Gemeinden und Regionen ausblendet und einen Rückzug des Staates propagiert. Auf Deutschland bezogen haben Monstadt und Scheiner (2016) gezeigt, wie die räumlichen Ausgangsvoraussetzungen der Bundesländer in unterschiedliche finanzielle Interessen übersetzt wurden. Weiterhin sind regionale Ungleichheiten bei Netzentgelten zu nennen, denen zufolge Verbraucher in dünn besiedelten Regionen mit einem hohen Anteil erneuerbarer Energieträger einen höheren Strompreis zahlen (Bundesnetzagentur, 2015). Auf lokaler Ebene können Renovierungsprojekte für eine effiziente Energienutzung dazu führen, dass energetische Sanierungen Gentrifizierungsprozesse befördern (Holm, 2011).

Zweitens sind neue, stärker regional geprägte Scales der Energieversorgung auch mit neuen Eigentumsstrukturen und Finanzierungskonzepten verbunden (Klagge und Anz, 2014). Die Gründung von lokalen oder regionalen Energiegenossenschaften kann nicht nur als eine Form der Dezentralisierung der Energieversorgung, sondern durchaus auch als eine neue Form der Privatisierung verstanden werden, da das Eigentum an Netzen und Anlagen - im Gegensatz zu kommunalem Eigentum - einen Mitgliedsbeitrag der GenossInnen erfordert. Haas und Sander (2013) beschreiben die Entstehung einer ,grünen Kapitalfraktion“, die in erneuerbaren Energien, unter anderem aufgrund garantierter Einspeisevergütungen, eine lukrative Anlagemöglichkeit sieht. Die Neugründung von Energiegenossenschaften zeigt erhebliche regionale Unterschiede dahingehend, dass Neugründungen in der Vergangenheit vor allem in den ländlichen Regionen Bayerns, Baden-Württembergs und Niedersachsens erfolgten, jedoch kaum in den neuen Bundesländern (Holstenkamp und Müller, 2013). Eine ähnliche Entwicklung zeigen auch Rekommunalisierungen von Energieversorgungsunternehmen, die für Städte und Gemeinden mit angespannter finanzieller Situation deutlich schwieriger zu realisieren sind. Die Regionalisierung bzw. Lokalisierung der Energieversorgung kann 
also mit der Entstehung neuer bzw. der Verstärkung bisheriger räumlicher Disparitäten auf unterschiedlichen Maßstabsebenen verbunden sein.

Drittens ist auch das Verhältnis zwischen sozialen Bewegungen und räumlicher Entwicklung in der Energiewende vielschichtig. So ist der Protest gegen Windenergieanlagen häufig mit der Frage verknüpft, wem die Gewinne aus den Anlagen zufließen. Lokale finanzielle und politische Beteiligung werden dabei als Voraussetzung für die Akzeptanz von Anlagen genannt (Gross, 2007). Die Proteste gegen Kohletagebaue treffen hingegen auf eine geteilte Akzeptanz unter der regionalen Bevölkerung. Schließlich ist der Kohleausstieg eng mit der Frage verknüpft, welche alternativen wirtschaftlichen Standbeine für Kohleregionen gefunden werden können (für die Lausitz: Keppler et al., 2011). Diese Problemstellung reibt sich an den ungleich verteilten globalen Auswirkungen der hier produzierten Emissionen, die vor allem ärmere Staaten des globalen Südens betreffen würden. Insofern schreibt sich ungleiche Entwicklung auf regionaler wie globaler Ebene in diese lokalen Proteste ein.

Diese Punkte sollen nicht die Energiewende insgesamt in Frage stellen, die bei allen Schwierigkeiten eine historische Gelegenheit darstellt, die Ära der fossilen und nuklearen Energieversorgung zu beenden. Wir möchten aber mit den skizzierten Ambivalenzen deutlich machen, dass die Energiewende und die damit verbundenen räumlichen Veränderungen ein politisch umkämpftes Projekt sind, das von verschiedenen Akteuren auf unterschiedlichen Maßstabsebenen ausgehandelt wird. Die skalare Ordnung der Energiewende ist daher von einer starken Fragmentierung mit teilweise auch widersprüchlichen Entwicklungen gekennzeichnet. Es ist aber gerade diese Widersprüchlichkeit, die einen Bezug der Scale-Perspektive auf die Energiewende wie auch energiegeographische Fragen insgesamt lohnend macht.

\section{Zusammenfassung und Ausblick}

Der Blick der Scale-Debatte auf die Konstruktion von räumlichen Maßstabsebenen, deren Wandel und Zusammenspiel sowie den strategischen Umgang mit ihnen, zeigt eindrücklich, wie gesellschaftliche Verhältnisse räumliche Strukturen prägen und gleichermaßen von diesen geprägt sind. Für die wechselseitige Durchdringung gesellschaftlicher und skalarer Ordnungen bieten der deutsche Energiesektor sowie dessen Transformation zahlreiche Beispiele. Die aktuellen materiellen, institutionellen und diskursiven Veränderungen in der Energieversorgung gehen einher mit der Entstehung neuer räumlicher Maßstabsebenen, dem Bedeutungswandel bestehender Scales und jeweils spezifischen skalaren Strategien von energiepolitischen Akteuren. Skalare Konfigurationen sind nicht nur Ausdruck gesellschaftlicher Kräfteverhältnisse, sondern auch Ergebnis gesellschaftlicher Auseinandersetzung. Mit dem Blick auf die Entstehung verschiedener räumlicher Ebenen geht die Scale-Debatte über die Multi-Level-
Perspektive der Transition Theory wie auch der politikwissenschaftlichen Arbeiten zu Multi-Level-Governance hinaus und liefert wertvolle Anregungen für eine kritische Energiegeographie. Die Frage nach den Maßstabsebenen der Energieversorgung und deren Neuordnung thematisiert den politischen Charakter des Energiesektors, der mit räumlichen Ungleichheiten untrennbar verbunden ist. Zudem bietet der Ansatz der Politics of Scale einen analytischen Rahmen, um lokale und globale Entwicklungen sowie deren Wechselwirkungen zu erfassen. In Anschluss an Markus Wissens Kritik der Multi-Level-Governance (2011:82f.) kann eine ScalePerspektive damit gesellschaftliche Transformationen, ein breiteres Spektrum an Akteuren sowie ungleiche Machtverhältnisse in den Blick nehmen. Damit ergibt sich in vielfältiger Hinsicht weiterer Forschungsbedarf für die Untersuchung ungleicher Entwicklung entlang von Energiefragen.

Erstens sind empirische Untersuchungen notwendig, um zu zeigen, wie sich die Verzahnung unterschiedlicher Scales vollzieht. Eine besondere Herausforderung ergibt sich aus den Überlagerungen und Wechselwirkungen verschiedener Ebenen. Was bedeutet das Neben-, Mit- und Gegeneinander verschiedener räumlicher Ebenen für die Energieversorgung? Welche Möglichkeiten und Widersprüche ergeben sich aus der skalaren Dialektik der Energieversorgung, wie können „glokale“ Unternehmen oder soziale Bewegungen agieren?

Zweitens bietet die Scale-Debatte Ansatzpunkte, Fragen der ,post-political city“ (Swyngedouw, 2009) auf energiepolitische Fragen zu beziehen. Die „Entpolitisierung“ der Energieversorgung war ebenso an konkrete Projekte und Maßstabsebenen geknüpft wie die gegenwärtig festzustellende Repolitisierung von Energiefragen. Die Debatte, ob der Energiesektor lokal, national oder international organisiert werden soll, adressiert damit bestehende Machtverhältnisse und deren mögliche Veränderung.

Daran anschließend stellt sich drittens bei Forderungen nach Energiedemokratie oder Energiegerechtigkeit (Bickerstaff et al., 2013) die Frage danach, auf welcher räumlichen Maßstabsebene bzw. mit welchen skalaren Strategien diese realisiert werden können. Machtverhältnisse im Energiesektor sind jedoch ganz wesentlich durch Scales geprägt. So zeigen erste Untersuchungen, dass es deutlich mehr lokale Initiativen für Energiedemokratie gibt als auf anderen, darüber hinausgehenden Ebenen (Becker und Kunze, 2014).

Die Perspektive der Scale-Debatte macht die Dynamik räumlicher Verhältnisse und deren Veränderbarkeit deutlich. Dies lässt sich anschaulich auf den Energiesektor übertragen, dessen technologische wie institutionelle Verfasstheit noch vor einigen Jahren als außerordentlich beharrlich galt und der sich aktuell in einer umfassenden Transformation befindet.

Datenverfüg barkeit. Für diesen Artikel wurden keine Datensätze genutzt. 
Interessenkonflikt. Die Autoren erklären, dass kein Interessenkonflikt besteht.

Danksagung. Vorarbeiten zu dem Beitrag entstanden im Rahmen des Projektes „Rescaling Environmental Governance in Europe“, gefördert von der Deutschen Forschungsgemeinschaft. Wir danken Ramona Bunkus für ihren Beitrag an Vorarbeiten für diesen Aufsatz. Darüber hinaus bedanken wir uns bei Benedikt Korf sowie den beiden anonymen GutachterInnen für die konstruktiven Hinweise.

Edited by: Benedikt Korf

Reviewed by: two anonymous referees

\section{Literatur}

Adger, N., Brown, K. und Thompson, E.: The Political Economy of Cross-Scale Networks in Resource Co-management, Ecol. Soc., 10, online, 2005.

Antentas, J.: Sliding Scale of Spaces and Dilemmas of Internationalism, Antipode, 47, 1101-1120, https://doi.org/10.1111/anti.12169, 2015.

Bauer, H.: Zukunftsthema „Rekommunalisierung“, Öffentliche Verwalt., 65, 329-338, 2012.

Bauriedl, S.: Formen lokaler Governance für eine dezentrale Energiewende, Geogr. Z., 104, 72-91, 2016.

Becker, S. und Kunze, C.: Transcending community energy: collective and politically motivated projects in renewable energy (CPE) across Europe, People, Place and Policy, 8, 180-191, https://doi.org/10.3351/ppp.0008.0003.0004, 2014.

Becker, S., Blanchet, T. und Kunze, C.: Social movements and urban energy policy: assessing contexts, agency and outcomes of remunicipalisation processes in Hamburg and Berlin, Util. Policy, 41, 228-236, https://doi.org/10.1016/j.jup.2016.02.001, 2016.

Becker, S., Gailing, L. und Naumann, M.: Neue Energielandschaften - Neue Akteurslandschaften, Eine Bestandsaufnahme im Land Brandenburg, Rosa-Luxemburg-Stiftung, Berlin, 2012.

Benz, A.: Multi-Level Governance, in: Handbuch Governance: Theoretische Grundlagen und empirische Anwendungsfelder, Herausgeber: Benz, A., Lütz, U. und Schimank, U., Wiesbaden, Nomos, 297-310, 2007.

Bickerstaff, K., Walker, G. und Bulkeley, H. (Hrsg.): Energy Justice in a Changing Climate, Social equity and low-carbon energy, Zed Books, London, 2013.

Bontrup, H. und Marquardt, R.: Kritisches Handbuch der deutschen Elektrizitätswirtschaft: Branchenentwicklung - Unternehmensstrategien - Arbeitsbeziehungen, edition sigma, Berlin, 2010.

Bosch, S., Rathmann, J., and Simetsreiter, F.: Raumverträglicher Ausbau von erneuerbaren Energien - ein alternativer Standortplanungsansatz für eine nachhaltige Energiewende, Geogr. Helv., 71, 29-45, https://doi.org/10.5194/gh-71-29-2016, 2016.

Bouzarovski, S., Bradshaw, M. und Wochnik, A.: Making territory through infrastructure: The governance of natural gas transit in Europe, Geoforum, 64, 217-228, https://doi.org/10.1016/j.geoforum.2015.06.022, 2015.
Brad, A.: Politische Ökologie und Politics of Scale - Vermittlungszusammenhänge zwischen Raum, Natur und Gesellschaft, Geogr. Helv., 71, 353-363, https://doi.org/10.5194/gh-71-353-2016, 2016.

Brenner, N.: New State Spaces. Urban Governance and the Rescaling of Statehood, Oxford University Press, Oxford, 2004.

Bridge, G., Bouzarovski, S., Bradshaw, M. und Eyre, N.: Geographies of energy transition: Space, place and the low-carbon economy, Energ. Policy, 53, 331-340, https://doi.org/10.1016/j.enpol.2012.10.066, 2013.

Brown, C. und Purcell, M.: There's nothing inherent about scale: Political ecology, the local trap, and the politics of development in the Brazilian Amazon, Geoforum, 36, 607-624, https://doi.org/10.1016/j.geoforum.2004.09.001, 2005.

Brücher, W.: Energiegeographie. Wechselwirkung zwischen Ressourcen, Raum und Politik, Borntraeger, Berlin/Stuttgart, 2009.

Bulkeley, H.: Reconfiguring environmental governance. Towards a politics of scales and networks, Polit. Geogr., 24, 875-902, https://doi.org/10.1016/j.polgeo.2005.07.002, 2005.

Bundesnetzagentur: Bericht Netzentgeltsystematik Elektrizität, Bundesnetzagentur, Bonn, 2015.

Calvert, K.: From 'energy geography' to 'energy geographies': Perspectives on a fertile academic borderland, Prog. Hum. Geog., 40, 105-125, https://doi.org/10.1177/0309132514566343, 2016.

Catney, P., MacGregor, S., Dobson, A., Hall, S., Royston, S., Robinson, Z., Ormerod, M. und Ross, S.: Big society, little justice? Community renewable energy and the politics of localism, Local Environment, 19, 715-730, https://doi.org/10.1080/13549839.2013.792044, 2014.

Chapura, M.: Scale, causality, complexity and emergence, rethinking scale's ontological significance, T. I. Brit. Geogr., 34, 462474, https://doi.org/10.1111/j.1475-5661.2009.00356.x, 2009.

Chatterton, P., Featherstone, D. und Routledge, P.: Articulating Climate Justice in Copenhagen: Antagonism, the Commons, and Solidarity, Antipode, 45, 602-620, https://doi.org/10.1111/j.1467-8330.2012.01025.x, 2013.

Coenen, L., Benneworth, P. und Truffer, B.: Toward a spatial perspective on sustainability transitions, Res. Policy, 41, 968-979, https://doi.org/10.1016/j.respol.2012.02.014, 2012.

Cohen, A. und Bakker, K.: The eco-scalar fix: rescaling environmental governance and the politics of ecological boundaries in Alberta, Canada, Environ. Plann. D, 32, 128-146, https://doi.org/10.1068/d0813, 2014.

Cohen, A. und McCarthy, J.: Reviewing rescaling: Strengthening the case for environmental considerations, Prog. Hum. Geog., 39, 3-25, https://doi.org/10.1177/0309132514521483, 2015.

Cowell, R., Ellis, G., Sherry-Brennan, F., Strachan, P. und Toke, D.: Rescaling the Governance of Renewable Energy: Lessons from the UK Devolution Experience, J. Environ. Pol. Plan., https://doi.org/10.1080/1523908X.2015.1008437, online first, 2015.

Dietz, K., Engels, B. und Pye, O.: Territory, scale and networks: The spatial dynamics of agrofuels, in: The Political Ecology of Agrofuels, Herausgeber: Dietz, K., Engels, B., Pye, O. und Brunnengräber, A., Routledge, Abingdon, 34-52, 2015.

Eiselt, J.: Dezentrale Energiewende: Chancen und Herausforderungen, Vieweg+Teubner, Wiesbaden, 2012.

Featherstone, D., Ince, A., MacKinnon, D., Strauss, K. und Cumbers, A.: Progressive localism and the construction of 
political alternatives, T. I. Brit. Geogr., 37, 177-182, https://doi.org/10.1111/j.1475-5661.2011.00493.x, 2012.

Freytag, T., Gössling, S. und Mössner, S.: Living the green city: Freiburg's Solarsiedlung between narratives and practices of sustainable urban development, Local Environment, 19, 644-659, https://doi.org/10.1080/13549839.2013.868872, 2014.

Fromme, J.: Energiesystemtransformation - räumliche Politik und Stromnetzplanung, Raumforsch. Raumordn., 74, 229-242, https://doi.org/10.1007/s13147-016-0402-y, 2016.

Gailing, L. und Röhring, A.: Was ist dezentral an der Energiewende? Infrastrukturen erneuerbarer Energien als Herausforderungen und Chancen für ländliche Räume, Raumforsch. Raumordn., 73, 31-43, https://doi.org/10.1007/s13147-014-0322-7, 2015.

Geels, F.: Technological transitions as evolutionary reconfiguration process: A multi-level perspective and a case-study, Res. Policy, 31, 1257-1274, https://doi.org/10.1016/S0048-7333(02)000628, 2002.

Geels, F. und Schot, J.: Typology of sociotechnical transition pathways, Res. Policy, 36, 399-417, https://doi.org/10.1016/j.respol.2007.01.003, 2007.

Goodman, M., Boykoff, M. und Evered, K.: Introduction: contentious geographies: environment, meaning, scale, in: Contentious Geographies: Environment, Meaning, Scale, Herausgeber: Goodman, M., Boykoff, M. und Evered, K. Ashgate, Aldershot, 1-26, 2008.

Gross, C.: Community perspectives of wind energy in Australia: the application of a community fairness framework to increase social acceptance, Energ. Policy, 35, 2727-2736, https://doi.org/10.1016/j.enpol.2006.12.013, 2007.

Haas, T.: Die Energiewende unter dem Druck (skalarer) Kräfteverschiebungen. Eine Analyse des EEG 2.0, Prokla, 184, 365-382, 2016.

Haas, T. und Sander, H.: Grüne Basis. Grüne Kapitalfraktionen in Europa - eine empirische Untersuchung, Rosa-LuxemburgStiftung, Berlin, 2013.

Harvey, D.: Justice, Nature \& the Geography of Difference, Blackwell, Oxford, 1996.

Heeg, S.: The erosion of corporatism? Rescaling of industrial relations in Germany, Eur. Urban Reg. Stud., 21, 146-160, https://doi.org/10.1177/0969776412445724, 2014.

Hoogesteger, J. und Verzijl, A.: Grassroots scalar politics: Insights from peasant water struggles in the Ecuadorian and Peruvian Andes, Geoforum, 62, 13-23, https://doi.org/10.1016/j.geoforum.2015.03.013, 2015.

Holm, A.: Ein ökosoziales Paradox. Stadtumbau und Gentrifizierung, politische ökologie, 124, 45-52, 2011.

Holstenkamp, L. und Müller, J. R.: Zum Stand von Energiegenossenschaften in Deutschland. Ein statistischer Überblick zum 31.12.2012, Leuphana-Universität, Lüneburg, http: //www.leuphana.de/fileadmin/user_upload/PERSONALPAGES/ _ijkl/janner_steve/Homepage_Master/wpbl_14.pdf, 2013.

Huber, M.: Theorizing energy geographies, Geography Compass, 9 , 327-338, https://doi.org/10.1111/gec3.12214, 2015.

Jessop, B.: Avoiding traps, rescaling the state, governing Europe, in: Leviathan Undone? Towards a Political Economy of Scale, Herausgeber: Mahon, R. und Keil, R., UBC Press, Vancouver, 87-104, 2009.
Jonas, A.: Pro scale, further reflections on the 'scale debate' in human geography, T. I. Brit. Geogr., 31, 399-406, https://doi.org/10.1111/j.1475-5661.2006.00210.x, 2006.

Keppler, D., Nölting, B. und Schröder, C. (Hrsg.): Neue Energie im Osten - Gestaltung des Umbruchs. Perspektiven für eine zukunftsfähige sozial-ökologische Energiewende, Frankfurt a.M. u.a., Peter Lang, 2011.

Klagge, B.: Governance-Prozesse für erneuerbare Energien - Akteure, Koordinations- und Steuerungsstrukturen, in: GovernanceProzesse für erneuerbare Energien, Herausgeber: Klagge, B. und Arbach, C., Akademie für Raumforschung und Landesplanung, Hannover, 7-16, 2013.

Klagge, B. und Anz, J.: Finanzialisierung der Windenergienutzung in Deutschland?, in: Politische Ökonomie der Finanzialisierung, Herausgeber: Heires, M. und Nölke, A., Springer VS, Wiesbaden, 241-257, 2014.

Klagge, B. und Brocke, T.: Decentralized electricity generation from renewable sources as a chance for local economic development: a qualitative study of two pioneer regions in Germany, Energ. Sustain. Soc., 2, 5, https://doi.org/10.1186/2192-0567-25, 2012.

Köhler, B.: Die Materialität von Rescaling-Prozessen. Zum Verhältnis von Politics of Scale und Political Ecology, in: Politics of Scale: Räume der Globalisierung und Perspektiven emanzipatorischer Politik, Herausgeber: Wissen, M., Röttger, B. und Heeg, S., Westfälisches Dampfboot, Münster, 208-223, 2008.

Lawhon, M. und Murphy, J.: Socio-technical regimes and sustainability transitions: Insights from political ecology, Prog. Hum. Geog., 36, 354-378, https://doi.org/10.1177/0309132511427960, 2012.

Lormes, I.: Kommunalisierung der Energieversorgung. Eine explorative Untersuchung von Stadtwerke-Gründungen, Springer VS, Wiesbaden, 2016.

MacKinnon, D.: Reconstructing scale: towards a new scalar politics, Prog. Hum. Geog., 35, 21-36, https://doi.org/10.1177/0309132510367841, 2011.

Marston, S., Jones, J. und Woodward, K.: Human geography without scale, T. I. Brit. Geogr., 30, 416-432, https://doi.org/10.1111/j.1475-5661.2005.00180.x, 2005.

Monstadt, J. und Scheiner, S.: Die Bundesländer in der nationalen Energie- und Klimapolitik: Räumliche Verteilungswirkungen und föderale Politikgestaltung der Energiewende, Raumforsch. Raumordn., 74, 179-197, https://doi.org/10.1007/s13147-0160395-6, 2016.

Norman, E., Bakker, K. und Cook, C.: Introduction to the Themed Section: Water Governance and the Politics of Scale, Water Alternatives, 5, 52-61, 2012.

Osofsky, H.: The Role of Climate Change Litigation in Establishing the Scale of Energy Regulation, Ann. Assoc. Am. Geogr., 101, 775-782, https://doi.org/10.1080/00045608.2011.567940, 2011.

Pütz, M.: Regional Governance. Theoretisch-konzeptionelle Grundlagen und eine Analyse nachhaltiger Siedlungsentwicklung in der Metropolregion München, oekom, München, 2005.

Radtke, J.: Bürgerenergie in Deutschland. Partizipation zwischen Gemeinwohl und Rendite, Springer VS, Wiesbaden, 2016.

Raven, R., Schot, J. und Berkhout, F.: Space and scale in sociotechnical transitions, Environmental Innovation and Societal Transitions, 4, 63-78, https://doi.org/10.1016/j.eist.2012.08.001, 2012. 
Reck, H.-J.: Rekommunalisierung. Modelle einer bürgernahen, nachhaltigen Energieversorgung, RaumPlanung, 158/159, 243 248, 2012.

Reed, M. und Bruyneel, S.: Rescaling environmental governance, rethinking the state: a three-dimensional review, Prog. Hum. Geog., 34, 646-653, https://doi.org/10.1177/0309132509354836, 2010.

Sander, H.: Die Bewegung für Klimagerechtigkeit und Energiedemokratie in Deutschland. Eine historisch-materialistische Bewegungsanalyse, Prokla, 184, 403-422, 2016.

Seyfang, G., Park, J. J. und Smith, A.: A thousand flowers blooming? An examination of community energy in the UK, Energ. Policy, 61, 977-989, https://doi.org/10.1016/j.enpol.2013.06.030, 2013.

Smith, N.: Homeless/global, scaling places, in: Mapping the Futures. Local Cultures, Global Change, Herausgeber: Bird, J., Routledge, London, 87-119, 1993.

Smith, N.: Scale, in: The Dictionary of Human Geography, 4. Auflage, Herausgeber: Johnston, R. J., Gregory, D., Pratt, G. und Watts, M., Blackwell, Oxford, 724-727, 2000.

Smith, N.: Uneven Development. Nature, Capital, and the Production of Space, 3. Auflage, University of Georgia Press, Athens, 2008[1984].

Späth, P. und Rohracher, H.: 'Energy regions': The transformative power of regional discourses on socio-technical futures, Res. Policy, 39, 449-458, https://doi.org/10.1016/j.respol.2010.01.017, 2010.

Späth, P. und Rohracher, H.: Beyond localism: The spatial scale and scaling in energy transitions, in: Scale-Sensitive Governance of the Environment, Herausgeber: Padt, F., Opdam, P., Polman, N. und Termeer, C., Wiley-Blackwell, Oxford, 106-121, 2014.
Stegert, P. und Klagge, B.: Akzeptanzsteigerung durch Bürgerbeteiligung beim Übertragungsnetzausbau? Theoretische Überlegungen und empirische Befunde, Geogr. Z., 103, 171-190, 2015.

Swyngedouw, E.: Technonatural revolutions: the scalar politics of Franco's hydro-social dream for Spain, 1939-1975, T. I. Brit. Geogr., 32, 9-28, https://doi.org/10.1111/j.14755661.2007.00233.x, 2007.

Swyngedouw, E.: The Antinomies of the Postpolitical City, in: Search of a Democratic Politics of Environmental Production, Int. J. Urban Regional, 33, 601-620, https://doi.org/10.1111/j.1468-2427.2009.00859.x, 2009.

Swyngedouw, E. und Heynen, N.: Urban Political Ecology, Justice and the Politics of Scale, Antipode, 35, 898-918, https://doi.org/10.1111/j.1467-8330.2003.00364.x, 2003.

von Bock und Polach, C., Kunze, C., Maaß, O. und Grundmann, P.: Bioenergy as a socio-technical system: The nexus of rules, social capital and cooperation in the development of bioenergy villages in Germany, Energy Research \& Social Science, 6, 128 135, https://doi.org/10.1016/j.erss.2015.02.003, 2015.

Wallerstein, I.: World-System Analysis: An Introduction, Duke University Press, Durham/London, 2004.

Wissen, M.: Zur räumlichen Dimensionierung sozialer Prozesse. Die Scale-Debatte in der angloamerikanischen Radical Geography - eine Einleitung, in: Politics of Scale: Räume der Globalisierung und Perspektiven emanzipatorischer Politik, Herausgeber: Wissen, M., Röttger, B. und Heeg, S., Westfälisches Dampfboot, Münster, 8-32, 2008.

Wissen, M.: Gesellschaftliche Naturverhältnisse in der Internationalisierung des Staates. Konflikte um die Räumlichkeit staatlicher Politik und die Kontrolle natürlicher Ressourcen, Westfälisches Dampfboot, Münster, 2011. 